\title{
Tetrahydrobiopterin Deficiency: From Phenotype to Genotype
}

\author{
Blau, Nenad ; Thöny, Beat ; Heizmann, Claus W ; Dhondt, Jean-Louis
}

\begin{abstract}
As a result of the selective screening worldwide during the last 18 years, approximately 250 patients with tetrahydrobiopterin deficiency were discovered. Most patients suffer from 6-pyruvoyl tetrahydropterin synthase deficiency (58\%), followed by dihydropteridine reductase deficiency (35\%), GTP cyclohydrolase I deficiency (3\%), and "primapterinuria" (4\%). The patients can be treated with neurotransmitter precursors, as well as with tetrahydrobiopterin. However, data on long term treatment are still scarce and it is therefore of great value to investigate all newborns with even mild hyperphenylalaninemia. Cloning of the enzymes involved in the biosynthesis and regeneration of tetrahydrobiopterin makes them to be easily accessible for biochemical and biological studies. So far, all proteins expressed heterologous are active in E. coli. Cloning of the wild type gene and mutant analysis of patients allow the rapid identification of the defective gene on the molecular level
\end{abstract}

DOI: https://doi.org/10.1515/pteridines.1993.4.1.1

Posted at the Zurich Open Repository and Archive, University of Zurich

ZORA URL: https://doi.org/10.5167/uzh-155436

Journal Article

Published Version

Originally published at:

Blau, Nenad; Thöny, Beat; Heizmann, Claus W; Dhondt, Jean-Louis (1993). Tetrahydrobiopterin Deficiency: From Phenotype to Genotype. Pteridines, 4(1):1-10.

DOI: https://doi.org/10.1515/pteridines.1993.4.1.1 
Pteridines

Vol. 4, 1993, pp. 1-10

\title{
Review
}

\section{Tetrahydrobiopterin Deficiency: From Phenotype to Genotype*}

\author{
Nenad Blau ${ }^{\S}$, Beat Thöny ${ }^{\#}$, Claus W. Heizmann ${ }^{\#}$, and Jean-Louis Dhondt ${ }^{\dagger}$ \\ ${ }^{ \pm}$Division of Clinical Chemistry. University Children’s Hospital, Steinwiesstr. 75, CH-8032 Zurich, \\ Switzerland \\ ${ }^{\dagger}$ Centre Hospitalier Sanit-Philbert. Faculté Libre de Médecine, Lomme Cedex, France
}

(Received January 10, 1993)

\section{Summary}

As a result of the selective screening worldwide during the last 18 years, approximately 250 patients with tetrahydrobiopterin deficiency were discovered. Most patients suffer from 6-pyruvoyl tetrahydropterin synthase deficiency $(58 \%)$, followed by dihydropteridine reductase deficiency (35\%), GTP cyclohydrolase I deficiency (3\%), and "primapterinuria" (4\%). The patients can be treated with neurotransmitter precursors, as well as with tetrahydrobiopterin. However, data on long term treatment are still scarce and it is therefore of great value to investigate all newborns with even mild hyperphenylalaninemia. Cloning of the enzymes involved in the biosynthesis and regeneration of tetrahydrobiopterin makes them to be easily accessible for biochemical and biological studies. So far, all proteins expressed heterologous are active in $E$. coli. Cloning of the wild type gene and mutant analysis of patients allow the rapid identification of the defective gene on the molecular level.

Key words: Tetrahydrobiopterin, Deficiency, Hyperphenylalaninemia, Gene cloning, DNA.

\section{Introduction}

Early detection of tetrahydrobiopterin $\left(\mathrm{BH}_{4}\right)$ deficiency became essential soon after it was recognized that a number of patients with hyperphenylalaninemia (HPA) show progressive neurological illness despite treatment with a low-phenylalanine diet $(1,2)$. The disease was initially named "malignant phenylketonuria (PKU)" because some of these patients died early. The first patients with $\mathrm{BH}_{4}$ deficiency were described as "a genetic variant of PKU" (3) and were later characterized as suffering from a defect in the regeneration of $\mathrm{BH}_{4} . \mathrm{BH}_{4}$ is known to be the natural cofactor of three aromatic amino acid oxidase: phenylalanine-4-hydroxylase (PAH), tyrosine-3-hydroxylase, and tryptophan-5-hydroxylase. $\mathrm{PAH}$ is responsible for the conversion of phenylala-

\footnotetext{
$\S$ Author to whom correspondence should be addressed.

*Dedicated to Professor Max Viscontini on the occasion of his 80 th birthday.
}

nine to tyrosine; tyrosine-3-hydroxylase and tryptophan-5-hydroxylase are the rate-limiting enzymes in the biosynthesis of catecholamines and serotonin, respectively (4). Thus, any defect in the biosynthesis or regeneration of $\mathrm{BH}_{4}$ will result in a deficiency of biogenic amine neurotransmitters accompanied by HPA.

About 250 patients with $\mathrm{BH}_{4}$ deficiency were discovered world-wide in the last 18 years $(5,6)$. Because of the different clinical and biochemical severity, this group of diseases was originally named "atypical PKU”, a term which today is reserved for mild forms of PAH deficiency.

Although a wide range of metabolic defects may exist, the term "typical" or "atypical" form will be used according to the actual need to treat, or not to treat, the patients. 


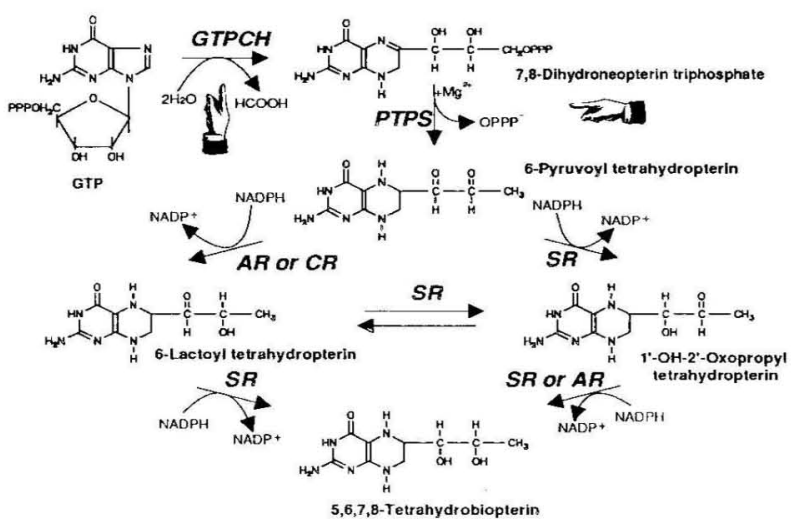

Figure 1. Biosynthesis of tetrahydrobiopterin including possible metabolic defects in hyperphenylalaninemia (1 is). GTPCH: GTP cyclohydrolase I: PTPS: 6-pyruvoyl tetrahydropterin synthase; SR: sepiapterin reductase; AR: aldose reductase: CR: carbonyl reductase.

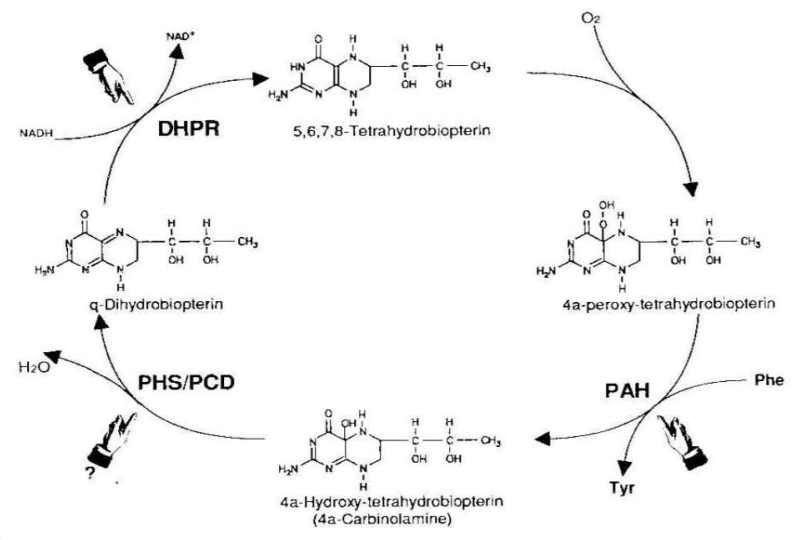

Figure 2. Phenylalanine hydroxylating system including possible metabolic defects in hyperphenylalaninemia (i) PAH: phenylalanine-4-hydroxylase: DHPR: dihydropteridine reductase: PHS/PCD: PAH stimulating protein/pterin-4a-carbinolamine dehydratase.

\section{Biochemical observation}

So far four molecular diseases have been known to cause HPA, including the classic form with a defect in the apoenzyme of PAH. Patients with the 3 variants suffer primarily from $\mathrm{BH}_{4}$ deficiency. either by one of the two defects in the biosynthesis (Fig. 1) or one in regeneration (Fig. 2) of the cofactor (7). They are all inherited autosomal recessive.

In GTP cyclohydrolase I (GTPCH) deficiency (8). the enzyme catalyzing the first step in the biosynthesis of $\mathrm{BH}_{4}$ is deficient and no pterins are synthesized. These patients are characterized by a neopterin and biopterin deficiency. Although it has been

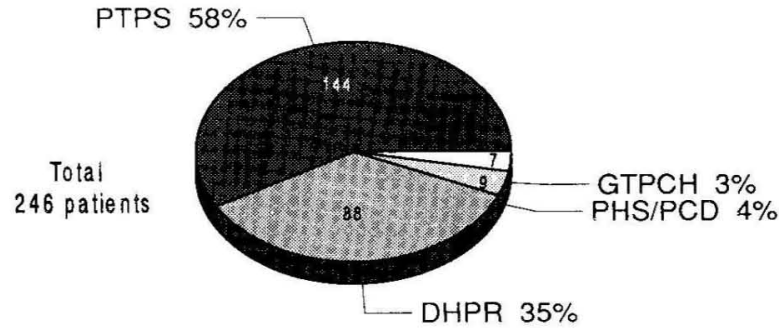

Figure 3. Results of the screening for tetrahydrobiopterin deficiency collected from the international register.

claimed that neopterin may play an active role in the cell-mediated immune response, cell proliferation, and differentiation, in one of these patients all humoral and cellular functions tested were found to be normal. In cerebrospinal fluid (CSF) neopterin, biopterin, isoxanthopterin, pterin, as well as the neurotransmitter metabolites, homovanillic acid (HVA) and 5-hydroxyindoleacetic acid (5HIAA), were generally low. This enzyme deficiency is rare, with 7 patients detected so far (Fig. 3).

6-Pyruvoyl-tetrahydropterin synthase (PTPS) deficiency (9) is the most common and heterogeneous variant with about 144 patients diagnosed all over the world, corresponding to about $58 \%$ of all patients with a $\mathrm{BH}_{4}$ deficiency (Fig. 3). Patients with severe, partial or peripheral, and transient forms have been reported (10). 7,8-Dihydroneopterin triphosphate can not be converted to 6-pyruvoyl tetrahydropterin (Fig. 1), resulting in an accumulation of 7,8-dihydroneopterin triphosphate in tissue of these patients. This intermediate is readily dephosphorylated by pyrophosphatase and excreted as dihydroneopterin and its oxidation product, neopterin. High concentrations of neopterin, monapterin (isomer of neopterin), and 3 -hydroxysepiapterin, and only traces of biopterin are found in the urine of the patients with PTPS deficiency. In servere (typical) forms of PTPS deficiency the neurotransmitter status in the CSF is similar to that in patients with GTPCH deficiency. In the peripheral or partial (atypical) forms of PTPS deficiency, normal levels of neurotransmitter metabolites are measured. It is therefore of practical importance to find out whether or not the patient belongs to this type of PTPS deficiency and thus may need different treatment (see below). The transient form of PTPS deficiency may be due to a maturation delay of $\mathrm{BH}_{4}$ biosynthesis.

In dihydropteridine reductase (DHPR) deficiency (11). the second most common form of $\mathrm{BH}_{4}$ deficiency (88 patients detected; Fig. 3), quinonoid-dihydrobiopterin formed during the hydroxylation of phenylalanine to tyrosine accumulates, and the 
amount of $\mathrm{BH}_{4}$ synthesized on the de novo pathway is insufficient for the normal function of hepatic PAH (Fig. 2). Similarly, as in the case of PTPS and GTPCH deficiencies, these patients are mostly characterized by a depletion of catecholamines and serotonin (12). Since quinonoid-dihydrobiopterin tautomerized readily to 7,8-dihydrobiopterin, patients with DHPR deficiency excrete high amounts of total biopterin (7,8-dihydrobiopterin and biopterin). In addition, owing to the lack of $\mathrm{BH}_{4}$ in these patients. there is no feedback inhibition of GTPCH and thus neopterin biosynthesis is generally activated.

Primapterinuria is the recently discovered variant of HPA characterized by the excretion of the 7-substituted pterins, primapterin (7-biopterin) and anapterin (7-neopterin) in the patient's urine $(13,14)$. Primapterinuria differs from classic PKU and other variants of $\mathrm{BH}_{4}$ deficiency because obviously these patients do not need any treatment. Nine patients have been detected so far (Fig. 3). The first patients were described by Dhondt et al. (15) and Blaskovics and Giudici (16). A loading test with $\mathrm{BH}_{4}$ was the first indication that primapterin may derive from biopterin (17). Furthermore, 7,8-dihydrobiopterin and sepiapterin given orally resulted in a significant increase in biopterin and primapterin, whereas the ratio remained between 1.0 and 1.5 after loading (18). From in vitro experiments it is known that primapterin can be formed during the $\mathrm{PAH}$ reaction in a phenylalanine-4-hydroxylase-stimulating protein (PHS)/pterin-4a-carbinolamine dehydratase (PCD) free system (Fig. 2) (19.20). So far, all known enzymes involved in the biosynthesis or regeneration of $\mathrm{BH}_{4}$ in these patients have been found to be normal and a deficiency of PHS/PCD has been proposed. However, PAH and PHS/PCD, per se, have not been measured.

\section{Clinical observation}

\section{Typical forms}

The clinical course of the illness in untreated patients is similar in typical (severe) PTPS, DHPR and GTPCH deficiencies (10). The median age at which clinical signs become evident is 4-5 months, but symptoms do not necessarily correlate with age of diagnosis, even in the same family. However, when information on the neonatal period is available, abnormal signs (poor sucking, decreased spontaneous movements, floppy baby) can be noticed during the neonatal period (6).

The common symptoms are mental retardation. convulsions (grand mal or myoclonic attacks), disturbances of tone and posture, drowsiness, irritability, abnormal movements, recurrent hyperthermia without infection, hypersalivation, swallowing difficulties. Diurnal fluctuation of alertness and neurologic symptoms are also reported. Microcephaly is observed in both diseases but with a higher incidence in PTPS deficiency (52\%) than in DHPR deficiency (33\%). However, in 13 out of 25 DHPR deficient patients for whom repeated measurements with increasing age were available, evolution of head circumference to progressive microcephaly was observed. whether patients were treated or not.

\section{Atypical forms}

Atypical PTPS deficiency: The absence of clinical signs theoretically defines atypical forms. However, in 2 cases neonatal hypotonia was noticed, 2 were mentally retarded, 1 had focal signs on the EEG, and 1 presented with acute but transient symptoms (behaviour problems, neurovegetative signs, sleeping difficulties).

Atypical DHPR deficiency: Some authors have suggested that partical deficiency of DHPR activity may also exist. That may explain a few reports on patients who were clinically unusual in some aspects (21). Primapterinuria: Although this form seems to have no serious consequences, minor abnormalities can be observed. In the first case (15), slight tremors of upper limbs after stimulations and a moderate tendency to hypertonia were noticed during the neonatal period. An EEG at 3 weeks of age revealed a few spikes and sharp waves in the frontal area. With dietary control of blood phenylalanine. neurological development normalized. In another case reported by Blaskovics and Guidici (16), transient hypotonia and motor delay were also noticed.

\section{Treatment}

The goals of the treatment are to control HPA by dietary restriction of phenylalanine or $\mathrm{BH}_{4}$ administration, and to restore neurotransmitter homeostasis by oral administration of amine precursors.

\section{Neurotransmitter replacement}

L-Dopa and 5-hydroxytryptophan (5-HT) administration represents a common therapeutic approach to $\mathrm{BH}_{4}$ deficiencies. Carbidopa, an inhibitor of peripheral aromatic amino acid decarboxylase, reduces the therapeutic dose of L-Dopa. 

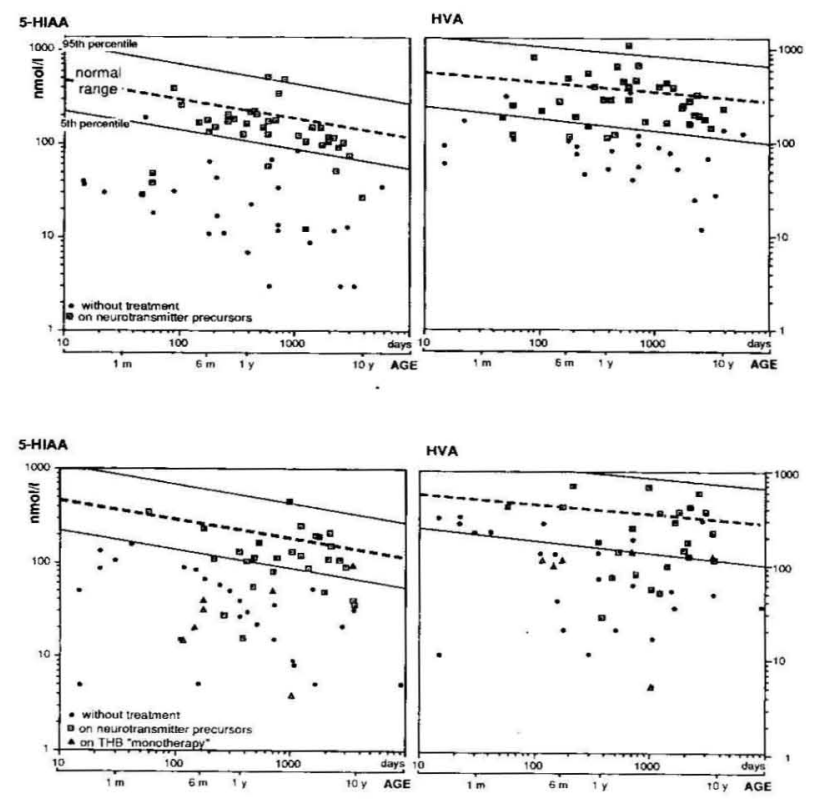

Figure 4. CSF levels of 5HIAA and HVA of patients with DHPR deficiency (upper) and PTPS deficiency (lower). without treatment: $\square$ on neurotransmitter precursors; $\triangle$ on $\mathrm{BH}_{4}$ "monotherapy": shaded area represents normal range (percentile 5-95) established from the distribution of 292 CSF determinations in normal confrols.

The monitoring of treatment represents the crucial point. Unfortuntely, there are no biochemical parameters measurable in the periphery which would allow the control of treatment. The analysis of neurotransmitter metabolites in CSF represents the only way to follow up the efficiency of treatment (Fig. 4). However, such an investigation cannot be easily repeated. Two cases (Fig. 5) illustrate the need of a balanced administration of Dopa and 5-HT.

In practice, the optimal dosage can only be found on a clinical basis and should be adjusted to the requirement of each patient with monitoring for adverse effects $(22,23)$ and disappearance of neurological symptoms. Diurnal fluctuations are often observed and may require changes in the schedule of drug administration. Difficulties with parents for compliance with treatment are frequently reported because of the constant need of treatment adjustments. which requires excellent collaboration with the family.

\section{Control of blood phenylalanine levels}

Although $\mathrm{BH}_{4}$ deficient persons exhibit a higher dietary phenylalanine tolerance than classical PKU patients, a limiting factor in the response to neurotransmitter precursor therapy might be the plasma
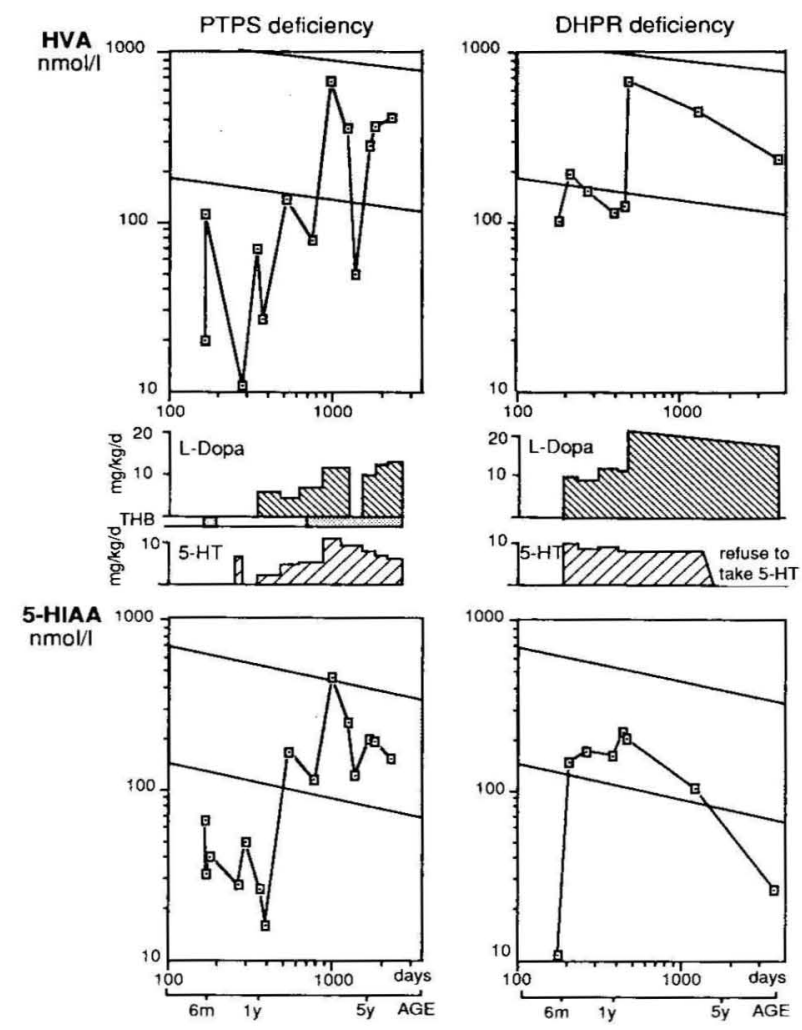

Figure 5. CSF monitoring of the treatment with neurotransmitter precursors.

phenylalanine fluctuations, which could alter the dose-effect relationships of these substances by interfering with their membrane transport or by competitive inhibition of tyrosine and tryptophan-hydroxylase (12). The control of blood phenylalanine concentrations has to be stricter than in other HPAs. Some patients on neurotransmitter treatment have had neurological problems with phenylalanine concentrations as low as $360 \mu \mathrm{mol} / l$.

\section{Tetrahydrobiopterin administration}

In PTPS deficiency, evidence of a bloc in $\mathrm{BH}_{4}$ synthesis invited attempts to treat these patients with $\mathrm{BH}_{4}$ replacement alone (so-called $\mathrm{BH}_{4}$ monotherapy). The main advantage was that $\mathrm{BH}_{4}$ replacement would not bypass any regulatory mechanisms that normally control neurotransmitter synthesis and secretion. However, in most cases $\mathrm{BH}_{4}$ monotherapy failed to restore a normal level of cerebral neurotransmitter synthesis as confirmed by low concentrations of 5HIAA and HVA in the CSF (Fig. 4). On the other hand, the use of $\mathrm{BH}_{4}$ to control blood phenylalanine levels appears to be efficient and is recommended. Relatively low doses of $\mathrm{BH}_{4}$ normal- 
ize blood phenylalanine levels and offer an interesting alternative to a phenylalanine-restricted diet.

In DHPR deficiency, $\mathrm{BH}_{4}$ cannot be given in sufficient amounts to maintain an adequate pool of $\mathrm{BH}_{4}$ in the absence of the endogenous reducing system sustained by DHPR.

\section{Folinic acid supplementation}

The hypothesis of a gradual CNS folate deficiency in DHPR deficienct patients is supported by low CSF folate levels, and also by the frequent occurrence of calcifications in the basal ganglia similar to those described in congenital folate malabsorption or in methotrexate toxicity. A direct benefit of folinic acid supplementation has been clinically demonstrated (24).

\section{The clinical outcome}

The replacement of neurotransmitters has been proven to be beneficial, though incomplete. Because in many cases the treatment was administered late, resolution of some of the major neurological signs gave the impression of a "remarkable" improvement, as initially published. Even when patients reached milestones for age, speech difficulties and sleeping problems were reported. Two PTPS and 6 DHPR deficient patients died although the treatment had been started within the first month of life.

Although results of a larger group of patients treated early might temper these pessimistic conclusions, four reasons can explain the limited success of treatment:

1. The possibility of fetal brain damage is likely to occur, especially in PTPS deficiency, since low birth weight, clinical signs, and microcephaly at birth are often reported.

2. Possible iatrogenic damage of neurotransmitter precursors cannot be excluded.

3. It can also be speculated that the quality of the response to therapy depends on the severity of the metabolic defect, as illustrated by the better response of patients with near normal CSF HVA concentrations at diagnosis (25). In DHPR deficiency, it has also been suggested that $\mathrm{CRM}^{+}$mutants may have a better prognosis (26).

\section{Do atypical forms have to be treated?}

These forms theoretically do not require treatment. Besides this affirmation, the decision to treat or not

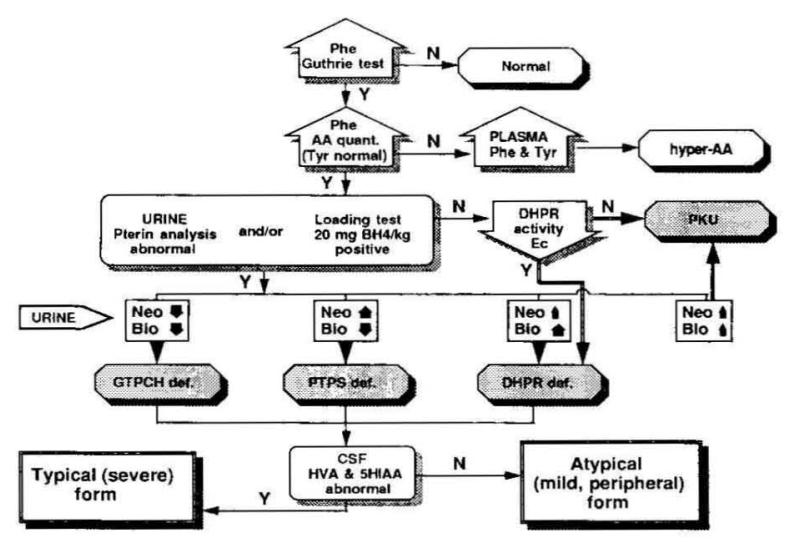

Figure 6. Screening policy and the diagnostic flow chart in the differentiation of hyperphenylalaninemia variants. 仓 represents increased value: \& represents deminished value. Phe: phenylalanine: Tyr: tyrosine: AA amino acids; Neo: neopterin: Bio: biopterin; $Y$ : yes; $N$ : no.

is not clear-cut. With regard to the obvious heterogeneity and unclear prognosis it seems adequate to treat newborns with the so called "atypical" or "peripheral" form, at least with $\mathrm{BH}_{4}$, and to monitor their development carefully.

\section{Screening for $\mathrm{BH}_{4}$ deficiency and differential diagno- sis}

Screening for $\mathrm{BH}_{4}$ deficiency should be done in all newborns with even slight hyperphenylalaninemia (plasma phenylalanine $<400 \mu \mathrm{mol} / l$ ) as well as in older children with neurological symptoms (27, 28). The following protocol should be followed:

1. Analysis of pterins in urine

2. Measurement of DHPR activity in blood from Guthrie cards

3. Analysis of phenylalanine in plasma before and after the $\mathrm{BH}_{4}$ loading test

The first two tests are essential and allow to differentiate between all variants of $\mathrm{BH}_{4}$ deficiency. With some limitations, the $\mathrm{BH}_{4}$ loading test is an additional useful diagnostic tool for the rapid discrimination between the classic PKU and biopterin variants. The diagnostic flow-chart is shown in Fig. 6.

\section{Analysis of pterins}

HPLC is the method which is used in most laboratories to analyze pterins in urine $(29,30)$. The analysis must be performed at elevated plasma phenylalanine levels and not under low-phenylalanine diet. Either a native urine or urine dried on filter 

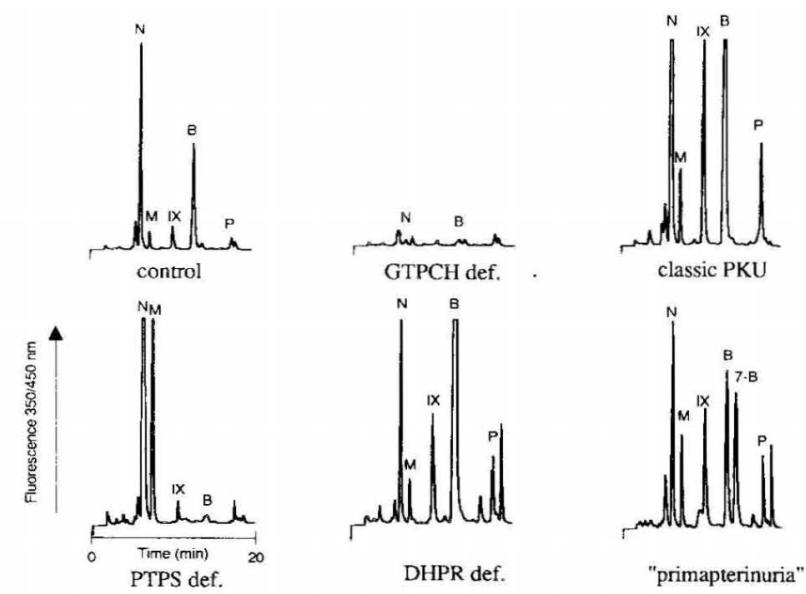

Figure 7. HPLC of urinary pterins (oxidized) from a healthy control and patients with different variants of hyperphenylalaninemia. $\mathrm{N}$ : neopterin; $\mathrm{M}$ : monapterin; $\mathrm{IX}$ : isoxanthopterin: $\mathrm{B}$ : biopterin: 7-B: primapterin; $\mathrm{P}$ : pterin.

paper (31) may be used for the selective screening. The concentrations of pterins are based on creatinine levels, which makes a urine collection unnecessary. A characteristic urinary pterin pattern makes it possible to identify all four variants of $\mathrm{BH}_{4}$ deficiency (Fig. 7).

In GTPCH deficiency, neopterin and biopterin excretion is extremely low (below $10 \%$ of normal) while the percentage of biopterin (of the sum of neopterin plus biopterin) is approximately $50 \%$ (neopterin/biopterin $\cong 1$ ). Patients with PTPS deficiency excrete trace amounts of biopterin and high amounts of neopterin (biopterin $<5 \%$; neopterin/biopterin $=$ $40-300$ ). Excretion of pterins is not always informative for DHPR deficiency. High biopterin concentrations (biopterin $>80 \%$ ) and normal or slightly increased neopterin concentrations are rather characteristic for older children suffering from DHPR deficiency and particularly in very young patients may be almost normal or close to values found in patients with classic PKU. In patients with primapterinuria the pterin pattern is similar to that in patients with PTPS deficiency (elevated neopterin and low biopterin; neopterin/biopterin $=18 \sim 36$ ) in the very early neonatal period. With increasing age neopterin normalizes while the excretion of primapterin persists and the ratio of biopterin to primapterin remains close to 1 .

\section{Loading test with $\mathrm{BH}_{4}$}

The loading test with $\mathrm{BH}_{4}$ allows the detection of all HPA variants with a defect of the $\mathrm{BH}_{4}$ biosynthesis, even if the defect is only partial. A rapid
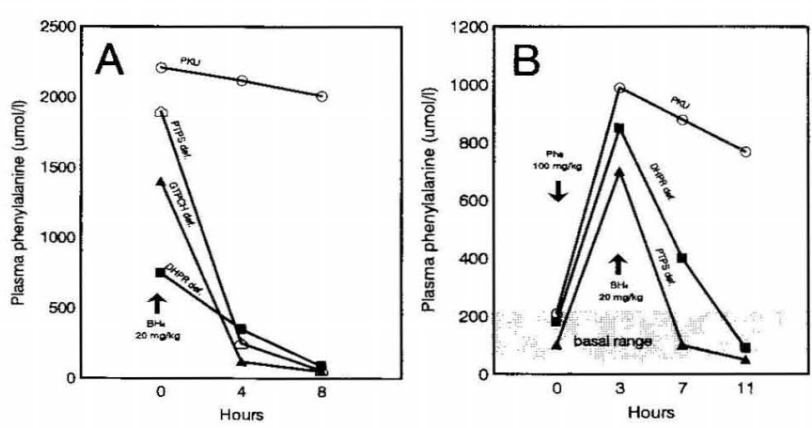

Figure 8. Typical results of a simple (A) and combined (B) tetrahydrobiopterin loading tests in patients with hyperphenylalaninemia.

normalization of the elevated plasma phenylalanine level occurs within 4 to 8 hours after administration of $\mathrm{BH}_{4}$ (Fig. 8A). The test was originally introduced by Niederwieser et al.(32) using $7.5 \mathrm{mg}$ of $\mathrm{BH}_{4} / \mathrm{kg}$ bw. However, it was soon noted that this procedure may fail in some patients with DHPR deficiency (26), namely those belonging to the $\mathrm{CRM}^{+}$(cross reactive material positive) type having a mutant enzyme. This limitation can be overcome by increasing the loading dose up to $20 \mathrm{mg} \mathrm{BH} / 4 \mathrm{~kg}$ bw. However one patient, described as a nonresponder even at high dose of $\mathrm{BH}_{4}$, died despite early diagnosis and treatment and may represent a "severe" form of DHPR deficiency (33).

An alternative procedure for the screening of hyperphenylalaninemiae is the combined phenylalanine $\left(100 \mathrm{mg} / \mathrm{kg}\right.$ bw) and $\mathrm{BH}_{4}(20 \mathrm{mg} / \mathrm{kg}$ bw) loading test (34). This test takes advantage of being performed while patients are on a phenylalanine-restricted diet, particularly when pterin analysis is not available. As for the conventional loading test, this also has to be combined with the measurement of DHPR activity in blood. The decrease of serum phenylalanine 4 and 8 hours after $\mathrm{BH}_{4}$ administration ( 7 and 11 hours after phenylalanine administration) allows differentiation between PTPS and DHPR deficiencies and classic PKU (Fig. 8B).

\section{Molecular biology of $\mathrm{BH}_{\mathbf{4}}$ biosynthetic and regenera- ting enzymes: gene cloning and expression}

Cloning of the genes or their corresponding cDNAs encoding the enzymes for $\mathrm{BH}_{4}$ metabolism will have many beneficial consequences such as the possibility to overproduce the proteins and purify them in large quantities for enzymatic and structural studies, and antibody production. In addition, for the defects described for the four enzymes involved 
in $\mathrm{BH}_{4}$ metabolism (see Figs. 1 and 2), identification of mutations on the DNA, responsible for aberrant expression or activity in patients, is the basis for future understanding of the deficiency on a molecular level.

GTPCH, the first biosynthetic enzyme for $\mathrm{BH}_{4}$ has been cloned and DNA sequence information is available from two different sources: rat liver (35) and Escherichia coli $(36,37)$. The isolated rat liver cDNA codes for a putative protein of 241 amino acids, whereas the mature protein has the first 11 amino acid residues deleted. Thus, posttranslational modification produces a protein of 230 amino acid residues with a molecular mass of 25,784 Da. Expression of the rat GTPCH cDNA in $E$. coli was reported to result in an active enzyme, indifferent whether it was expressed as the premature form (complete sDNA) or as the mature form (38). The mature $E$. coli GTPCH contains 222 amino acid residues with a molecular mass of $24,742 \mathrm{Da}$. For this enzyme, only the starting methionine was found to be removed. An amino acid alignment of the rat and $E$. coli sequences revealed homology extending over the entire length of the proteins. Overproduction and purification of recombinant GTPCH from $E$. coli allowed the crystallization and a preliminary characterization of the X-ray structure (39). Although a fragment coding for the human GTPCH was reported to be cloned (39), isolating of the complete coding region is eagerly awaited in order to study defects in patients with reduced GTPCH acitivity.

The PTPS is the most frequent enzyme acitivity that is reduced or missing in patients exhibiting a $\mathrm{BH}_{4}$ deficiency. The rat and human liver cDNAs have been cloned and sequenced $(40-42)$. The rat ¿DNA codes for a protein of 144 amino acids. The first four amino acids are not present in the purified enzyme, resulting in a mature rat protein with a molecular mass of 15,855 Da. The human liver cDNA codes for a protein of 145 residues with an estimated molecular weight of 16,387. It exhibits a $82 \%$ identity to the rat amino acid sequence. Since the amino terminus of the human PTPS was not accessible by Edman degradation due to end blocking, the N-terminus of the mature protein is not known. Both, the rat and human cDNA can be expressed as an active enzyme in $E$. coli that makes the PTPS easily accessible for further analysis. This powerful system made it possible to identify the first mutations in the coding region for PTPS, responsible for a lowered enzymatic activity in patients with $\mathrm{BH}_{4}$ deficiency (41, Thöny et al., unpublished).

A cDNA for SR, the enzyme catalyzing the final step in the $\mathrm{BH}_{4}$ biosynthesis. was isolated from rat and human liver $(43,44)$. The rat liver cDNA encodes a polypeptide of 262 amino acid residues with a molecular mass of $28,169 \mathrm{Da}$, including the $\mathrm{N}$-terminally acetylated methionine (45). A corresponding cDNA isolated from human liver codes for a protein of 261 amino acid residues with a molecular mass of 28,047 Da. A sequence comparison revealed a $74 \%$ identity between these two enzymes. The rat liver enzyme was expressed in $E$. coli as a translational fusion to the $\mathrm{N}$-terminal fragment of $\beta$-galactosidase. Since this resulted in an active SR, it is expected that also the human enzyme, expressed as a non-fusion protein in $E$. coli, will yield SR activity.

Alternative reductases to $\mathrm{SR}$, such as $\mathrm{AR}$ and $\mathrm{CR}$, were found to accomplish at least in vitro the two step reduction of the carbonyl groups (compare with Fig. 1; (46)). These alternative pathways might account in vivo for the fact that so far no SR deficiency was observed in man. Human cDNAs for AR and $\mathrm{CR}$ have been cloned from several tissues but will not be discussed in this article (47-50).

Following the oxidation of $\mathrm{BH}_{4}$ by the $\mathrm{PAH}$, the pterin cofactor is proposed to be efficiently recycled in the presence of two proteins, the PHS/PCD and DHPR (Fig. 2). Based on the elucidation of the complete amino acid sequence of the human and rat PHS/PCD (51), the corresponding cDNA was cloned from rat and human liver (Thöny et al., unpublished). The native protein was found to be identical in rat and human, and consists of 103 amino acids with a molecular mass of $11,909 \mathrm{Da}$, The Nterminally encoded methionine is cleaved off, and the second amino acid, the alanine residue, was found to be acetylated in the mature protein. Heterologous expression of the cDNA in $E$. coli yielded the activity in a crude extract, thus allowing the purification of the protein in large amounts and making it accessible to refined biochemical studies. Unexpectedly, the PHS/PCD was found to be identical to the hepatic nuclear dimerization factor for the transcription factor HNF-1 $\alpha$ (52, Thöny et al.. unpublished). To clarify the meaning of this finding, it will be hepful to have recombinant protein available, free from any contamination, to perform in vitro studies with PAH. In addition, the newly described form of hyperphenylalaninemia characterized by 7pterin excretion was proposed to be due to a defect in PHS/PCD activity (13-17). The isolation of the DNA encoding this protein will allow to further investigate the enzymatic defects in such patients on the molecular level.

The enzyme responsible for the completion of the reductive recycling of $\mathrm{BH}_{4}, \mathrm{DHPR}$, is thus far the protein best studied biochemically and genetically. 
The DNA sequence encoding the DHPR is available from rat and human liver (53-55). The rat liver cDNA codes for 241 amino acids. However, the mature protein appears to have the initiating methionine cleaved off and an acetylated alanine residue on its $\mathrm{N}$-terminus. The molecular weight of 25,420 ( 240 amino acids) is very similar to that of the human liver enzyme and a sequence alignment shows that the two proteins differ only in 10 amino acid residues. The human DHPR protein has a length of 244 amino acids and a molecular mass of 25,760 Da (serine at position 51, (ref. 54)) or 25,774 Da (threonine at position 51, (ref. 55)). The human enzyme was also reported to be N-terminally blocked due to acetylation, however, the mature end of the protein is not known. Heterologous expression of the entire cDNA encoding the human DHPR has been reported and yielded the active enzyme in E. coli (56). Moreover, the rat liver DHPR was crystallized and its 3-dimensional structure was elucidated (57). Interestingly, it is structurally and mechanistically different from the dihydrofolate reductase. Using PCR technology, mutations on the DNA level were detected that caused a change in the polypeptide chain $(21,58)$. Also, RFLP was shown to be a useful tool for prenatal diagnosis of DHPR deficiency (59).

In summary, it is remarkable that probably all the human metabolic enzymes for $\mathrm{BH}_{4}$ biosynthesis and recycling appear to be active when expressed in E. coli. Mutations in GTPCH, PTPS, PHS/PCD or DHPR can now be easily detected for their effects on enzyme activity be expressing them in $E$. coli. At the same time, the purification of native and also mutant enzymes in large amounts will be facilitated. Cells from patients or patients cultured fibroblasts contain insufficient material to isolate enzymes for such studies. Using PCR-based technology, prenatal diagnosis for $\mathrm{BH}_{4}$ deficiency might be introduced as a noninvasive method without any risk for the offspring. In the future, one might even consider a completely new area of treatment by using gene therapy, i.e. transferring the wild-type gene into patients with a $\mathrm{BH}_{4}$-deficiency in order to restore the defective gene. Such an approach was shown to be feasible in vitro for cells derived from patients with a defect in the DHPR gene (60).

\section{Acknowledgements}

The authors thank M. Killen for help with preparing the manuscript. This work was supported by the Swiss National Science Foundation, Grant No.
31-33897.92, by the Helmut Horten Research Foundation, and Jubiläumsspende der Universität Zürich.

\section{References}

1. Bartholomé $\mathrm{K}$. A new molecular defect in phenylketonuria. Lancet 1974; ii: 1580 .

2. Smith I. Atypical phenylketonuria accompanied by a severe progressive neurological illness unresponsive to dietary treatment. Arch Dis Childhood 1974; 49: 245.

3. Tada K. Yoshida T, Mochizuki K, et al. Two siblings of hyperphenylalaninemia: suggestion to a genetic variant of phenylketonuria. Tohoku J Exp Med 1969; 100: 249-53.

4. Kaufman S. Metabolism of the phenylalanine hydroxylation cofactor. J. Biol Chem 1961; 242: 3934-43.

5. Dhondt J-L. Register of tetrahydrobiopterin deficiency. Bagnolet: Milupa 1991; 7-11.

6. Dhondt JL. Tetrahydrobiopterin deficiencies. Lessons from the compilation of 200 patients. Developmental Brain Dysfunction 1993; 6: 139-55.

7. Blau N. Inborn errors of tetrahydrobiopterin metabolism. Ann Rev Nutr 1988; 8: 185-209.

8. Niederwieser A. Blau N, Wang M. et al. GTP cyclohydrolase I deficiency, a new enzyme defect causing hyperphenylalaninemia with neopterin, biopterin, dopamine, and serotonin deficiencies and muscular hypotonia. Eur J Pediatr 1984: 141: 208-14

9. Niederwieser A. Leimbacher W, Curtius HCh, et al. Atypical phenylketonuria with "dihydrobiopterin synthetase" deficiency: Absence of phosphate eliminating enzyme activity demonstrated in liver. Eur J Pediatr 1985; 144: 13-6.

10. Dhondt JL. Tetrahydropiopterin deficiencies. Preliminary analysis from an International Survey. J Pediatr 1984; 104: $501-8$

11. Kaufman S, Hotzman NA. Milstien S, Butler IJ, Krumholz A. Phenylketonuria due to a deficiency of dihydropteridine reductase. N Engl J Med 1975; 293: 785-90.

12. Ponzone A. Guardamagna O, Ferraris S. Biasetti S. Bracco G. Niederwieser A. Neurotransmitter therapy and diet in malignant phenylketonuria. Eur J Pediatr 1987; 146: 93-4.

13. Curtius HCh, Kuster Th, Matasovic A. Blau N. Dhondt J-L. Primapterin, anapterin and 6-oxo-primapterin, three new 7-substituted pterins identified in a patient with hyperphenylalaninemia. Biochem Biophys Res Commun 1988: 153: $715-21$.

14. Blau N. Dhondt J-L. Kuster Th. Curtius HCh. New variant of hyperphenylalaninemia with excretion of 7-substituted pterins Eur J Pediatr 1988; 148: 176.

15. Dhondt JL. Guibaud P. Rolland MO. Dorche C, Andre S. Forzy G. Hayte JM. Neonatal hyperphenylalaninemia presumably caused by a new variant of biopterin synthetase deficiency. Eur J Pediatr 1988: 147: 153-7.

16. Blaskovics M. Giudici TA. A new variant of biopterin deficiency. N Engl J Med 1988: 319: 1611-2.

17. Blau N. Curtius HCh. Kuster Th. et al. Primapterinuria: a new variant of atypical phenylketonuria. $\mathbf{J}$ Inher Metab Dis 1989: 12(Suppl 2): 335-8.

18. Giudici TA. Blaskovics M. Lim B. Gambetta R. Curtius $\mathrm{HCh}$. Blau N. Excretion of 7-substituted pterins by a hy- 
perphenylalaninemic variant (primapterinuria). Administration of tetrahydrobiopterin and sepiapterin. In: Blau $\mathrm{N}$, Curtius HCh, Levine RA, Cotton RGH, eds. Pterins and Biogenic Amines in Neurology, Pediatrics and Immunology 1991; 149-64. Grosse Pointe: Lakeshore Publishing Co.

19. Curtius HCh, Adler C, Rebrin I, Heizmann CW, Ghisla S. 7-Substituted pterins: Formation during phenylalanine hydroxylation in the absence of dehydratase. Biochem Biophys Res Commun 1990; 172: 1060-6.

20. Davis DM, Kaufman S, Milstien S. Conversion of 6-substituted tetrahydropterins to 7-isomers via phenylalanine hydroxylase-generated intermediates. Proc Natl Acad Sci 1991; 88: 385-9.

21. Blau N. Heizmann CW. Sperl W. et al. Atypical (mild) forms of dihydropteridine reductase deficiency: neurochemical evaluation and mutation detection. Pediatr Res 1992; 32: $726-30$.

22. McInnes RR, Kaufman S, Warsh JJ, et al. Biopterin synthesis defect. Treatment with L-dopa and 5-hydroxytryptophan compared with therapy with a tetrahydropterin. J Clin Invest 1984; 73: 458-69.

2: Tanaka Y, Matsuo N. Tsuzaki S. Araki K. Tsuchiya Y, Niederwieser A. On-off phenomenon in a child with tetrahydrobiopterin deficieny due to 6-pyruvoyl tetrahydropterin synthase deficiency. Eur J Pediatr 189: 148: 450-2.

24. Smith I, Hyland K, Kendall B, Leemin RJ. Clinical role of pteridine therapy in tetrahydrobiopterin deficiency. $\mathrm{J}$ Inher Metab Dis 1985; 8(suppl. 1): 34-8.

25. Giugliani R. Costa da Costa J, Dutra-Filho CS, Dutra JC. Pereira MLS, Niederwieser A. Successful therapy of hyperphenylalaninemia due to defective tetrahydrobiopterin metabolism in two siblings. Rev Brasil Genet 1986: 9: $685-92$

26. Ponzone A. Guardamagna O. Ferraris S. Bracco G. Niederwieser A, Cotton RGH. Two mutations of dihydropteridine reductase deficiency. Arch Dis Child 1988; 63: 1547.

27. Dhondt JL. Strategy for the screening of tetrahydrobiopterin deficiency among hyperphenylalaninemic patients: 15-years experience. J Inher Metab Dis 1991; 14: 117-27.

28. Blau N. Guidelines for the screening for hyperphenylalaninemia due to tetrahydrobiopterin deficiency. Cro Med J 1992; 33: 17-22.

29. Dhondt JL, Largilliére C. Ardouin P, Farriaux JP, Dautrevaux M. Diagnosis of variants of hyperphenylalaninemia by determination of pterins in urine. Clin Chim Acta 1981; 110: 205-15.

30. Niederwieser A. Staudenmann W. Wetzel E. High-performance liquid chromatograph with column switching for the analysis of biogenic amine metabolites and pterins. J Chromatogr 1984: 290: 237-46.

31. Blau N, Kierat L. Heizmann CW. Endres W, Giudici T. Wang M. Screening for tetrahydrobiopterin deficiency in newborns using dried urine on filter paper. J Inher Metab Dis 1992; 15: 402-4

32. Niederwieser A. Curtius HCh. Viscontini M, Schaub J. Schmidt H. Phenylketonuria variants. Lancet 1979: 1: 550.

33. Endres W. Ibel H, Kierat L, Blau N, Curtius HCh. Tetrahydrobiopterin and "non-responsive" dihydropteridine reductase deficiency. Lancet 1987: ii: 223.
34. Ponzone A, Guardamagna O. Spada M, et al. Differential diagnosis of hyperphenylalaninemia by a combined phenylalanine-tetrahydrobiopterin loading test. Eur J Pediatr 1993; in press.

35. Hatakeyama K Inoue Y, Harada T, Kagamiyama H. Cloning and sequencing of cDNA encoding rat GTP cyclohydrolase I. J Biol Chem 1991; 266: 765-9.

36. Katzenmeier G, Schmid C, Bacher A. Cloning and expression of the putative gene coding for GTP cyclohydrolase I from Escherichia coli. FEMS Microbiology Letters 1990; 66: $231-4$.

37. Katzenmeier G, Schmid C, Kellermann J, Lottspeich F. Bacher A. Sequence of GTP cyclohydrolase I from Escherichia coli. Biological Chemistry Hoppe-Seyler 1991; 372 : 991-7.

38. Hatakeyama K. Harada T, Kagamiyama H. Expression, purification and characterization of premature and mature forms of rat GTP cyclohydrolase I in Escherichia coli using pMal vector. In: Eds Blau N. Curtius HCh, Levine R. and Yim J. Seoul: Pteridines and Related Biogenic Amines and Folates. Hanrim Publishing Co., 1992; 107-8.

39. Schmid C, Ladenstein R, Luecke H. Huber R. Bacher, A. Crystallization and preliminary crystallographic characterization of GTP cyclohydrolase I from Escherichia coli. J Mol Biol 1992: 226: 1279-81.

40. Inoue Y. Kawasaki Y. Harada T, Hatakeyama K. Kagamiyama $\mathrm{H}$. Purification and cDNA cloning of rat 6-pyruvoyltetrahydropterin synthase. J Biol Chem 1991; 266: 207916.

41. Hatakeyama K. Ashida A, Owada M, Kitagawa T, Mino M. Kagamiyama, H. Molecular basis of malignant hyperphenylalaninemia: a mutation in the gene encoding human 6-pyruvoyl-tetrahydropterin synthase. In: Pteridines and Related Biogenic Amines and Folates. Eds: Blau N. Curtius, HCh, Levine R. and Yim J. Hanrim Publishing Co.. Seoul. 1992; 142-3.

42. Thöny B. Leimbacher W. Bürgisser D. Heizmann CW. Human 6-pyruvoyltetrahydropterin synthase: cDNA cloning and heterologous expression of the recombinant enzyme. Biochem Biophys Res Comm 1993; in press.

43. Citron BA. Milstien S, Gutierrez JC, Levine RA, Yanaka BL, Kaufman S. Isolation and expression of rat liver sepiapterin reductase cDNA. Proc Natl Acad Sci USA 1990: 87: $6436-40$

44. Ichinose H. Katho S, Sueoka T, Titani K. Fujita K. Nagatsu $T$. Cloning and sequencing of cDNA encoding human sepiapterin reductase. Biochem Biophys Res Comm. 1991; 179: $183-9$.

45. Oyama R, Katho S. Sueoka T. et al. The complete amino acid sequence of the mature form of rat sepiapterin reductase. Biochem Biophys Res Comm 1990; 173: 627-31.

46. Park YS. Heizmann CW, Wermuth B, et al. Human carbonyl and aldose reductases: new catalytic functions in tetrahydrobiopterin biosynthesis. Biochem Biophys Res Comm 1991: 175: 738-44.

47. Bohren KM. Bullock B, Wermuth B, Gabbay KH. The aldo-keto reductase superfamily. J Biol Chem 1989: 264: 9547-51.

48. Garcia-Perez A. Martin B. Murphy HR. et al. Molecular cloning of cDNA coding for kidney aldose reductase. J Biol Chem 1989: 264: 16815-21. 
49. Forrest GL, Akman S. Krutzik S, et al. Induction of human carbonyl reductase gene located on chromosome 21. Biochim Biophys Acta 1990; 1048: 149-85.

50. Nishimura C, Matsuura Y, Kokai Y, et al. Cloning and expression of human aldose reductase. J Biol Chem 1990: 265: $9788-92$.

51. Hauer CR, Rebrin I, Thöny B, et al. Phenylalanine hydroxylase-stimulating protein/pterin-4a-carbinolamine dehydratase from rat and human liver. J Biol Chem 1993; 268: in press.

52. Mendel D, Kahavari PA, Conley PB, et al. Characterization of a cofactor that regulates dimerization of a mammalian homeodomain protein. Science 1991; 254: 1762-7.

53. Shahbaz M. Hoch JA. Trach KA. Hural JA, Webber S. Whiteley JM. Structural studies and isolation of cDNA clones providing the complete sequence of rat liver dihydropteridine reductase. J Biol Chem 1987; 262: 16412-6.

54. Dahl HHM. Hutchison W. McAdam W. Wake S, Morgan FJ, Cotton RGH. Human dihydropteridine reductase: characterization of a cDNS clone and its use in analysis of patients with dihydropteridine reductase deficiency. Nucl Acids Res 1987; 15: 1921-32

55. Lockyer J, Cook RG, Milstien S, Kaufman S. Woo SLC,
Ledley FD. Structure and expression of human dihydropteridine reductase. Proc Natl Acad Sci USA 1987: 84: 332933.

56. Armarego WLF, Cotton RGH, Dahl HHM, Dixon NE. High-level expression of human dihydropteridine reductase (EC 1.6.99.7), without $\mathrm{N}$-terminal amino acid protection. in Escherichia coli. Biochem. J 1989; 261, 256-8.

57. Varughese Ik. Skinner MM. Whiteley JM. Matthews DA. Crystal structure of rat liver dihydropteridine reductase. Proc Natl Acad Sci USA 1992; 89: 6080-4.

58. Howells DW. Forrest SM. Dahl H-HM. Cotton RGH. Insertion of an extra codon for threonine is a cause of dihydropteridine reductase deficiency. Am J Hum Genet 1990; 47: $279-85$.

59. Hayasaka K. Narisiawa K. Ohura T, Ogawa E. Dahl HHM. Restriction fragment length polymorphisms among Japanese detected with a dihydropteridine reductase cDNA gene probe. J Inher Metab Dis 1990; 13: 873-8.

60. Mikami H, Matsubara Y, Hayasaka K, et al. Molecular analysis of dihydropteridine reductase deficiency and restoration of the enzyme activity by gene transfer. $J$ Inher Metab Dis 1990; 13: 787-91. 